\title{
The Significance of the Relative Lymphocyte Count as an Independent Predictor of Cardiovascular Disease in Patients with Obstructive Sleep Apnea Syndrome
}

\author{
Vildan Altunayoglu Cakmak ${ }^{a}$ Savas Ozsu $^{b}$ Ayhan Gulsoy ${ }^{b}$ Ramazan Akpinar ${ }^{a}$ \\ Yilmaz Bulbul ${ }^{\mathrm{b}}$
}

Departments of a Neurology and ${ }^{b}$ Pulmonary Medicine, Faculty of Medicine, Karadeniz Technical University,

Trabzon, Turkey

\section{Key Words}

Obstructive sleep apnea syndrome $\cdot$ Relative lymphocyte

count · Cardiovascular diseases · Inflammation

\begin{abstract}
Objective: The aim of this study was to determine whether or not relative lymphocyte count (RLC) is associated with cardiovascular disease (CVD) in patients with obstructive sleep apnea syndrome (OSAS). Subjects and Methods: In this study, 141 patients diagnosed with OSAS using polysomnography were enrolled. Patients were classified according to the severity of OSAS as determined by the apnea-hypopnea index (AHI) and presence of CVD. Lymphocyte count and other hematological parameters at complete blood count were determined and compared between patients with and without CVD. Multivariate regression analysis was used to estimate the associated factors for presence of CVD. Results: Absolute and relative lymphocyte counts were lower in the OSAS patients with CVD compared to those without CVD (mean absolute lymphocyte counts: $2.0 \times 10^{3}$ vs. $2.5 \times$ $10^{3} \mu \mathrm{l}, \mathrm{p}=0.004$, and mean RLC: 28.3 vs. $33.9 \%, \mathrm{p}=0.001$, respectively). OSAS patients with CVD (14.2) had higher red cell distribution width values than the patients without CVD
\end{abstract}

\begin{tabular}{ll}
\hline KARGER & $\begin{array}{l}\text { ( } 2016 \text { S. Karger AG, Basel } \\
1011-7571 / 16 / 0255-0455 \$ 39.50 / 0\end{array}$ \\
$\begin{array}{l}\text { E-Mail karger@karger.com } \\
\text { www.karger.com/mpp }\end{array}$ & $\begin{array}{l}\text { This is an Open Access article licensed under the terms of the } \\
\text { Creative Commons Attribution-NonCommercial 3.0 Un- } \\
\text { ported license (CC BY-NC) (www.karger.com/OA-license), } \\
\text { applicable to the online version of the article only. Distribu- } \\
\text { tion permitted for non-commercial purposes only. }\end{array}$
\end{tabular}

(13.4) $(p=0.005)$. Multivariate analysis identified RLC as an independent predictor of CVD in patients with OSAS (odds ratio $=0.9,95 \% \mathrm{Cl}: 0.85-1.0, p=0.042$ ). Conclusion: $\mathrm{RLC}$ was identified as an independent predictor of CVD in patients with OSAS. Since RLC is a widely available diagnostic tool with no additional costs over the routinely performed complete blood count, it can be used for predicting CVD in patients with OSAS.

(c) 2016 S. Karger AG, Basel

\section{Introduction}

Obstructive sleep apnea syndrome (OSAS) is a chronic disease characterized by repeated obstructions of the upper airway with oxyhemoglobin desaturation and brief arousals during sleep [1]. OSAS is commonly associated with atherosclerotic conditions such as cardiovascular and cerebrovascular diseases [1]. Although the underlying mechanism in this association is unclear, a chronic inflammatory state and endothelial dysfunction as a consequence of intermittent hypoxia and fragmented sleep are thought to play important roles in the OSAS pathogenesis [2]. 
Several routine complete blood count (CBC) parameters may provide important clues about diseases that involve inflammatory and coagulation processes [3]. The value of these parameters in risk and prognostic evaluation of cardiovascular disease (CVD) were reported in several studies that evaluated the leukocyte, mean platelet volume (MPV), platelet-to-lymphocyte ratio (PLR), red cell distribution width (RDW), and neutrophil-to-lymphocyte ratio [4-8]. An association was previously reported between a low lymphocyte count, especially a low relative lymphocyte count (RLC), and poor prognosis in CVD [9-11].

In patients with OSAS, CBC parameters such as MPV $[12,13], \mathrm{RDW}[14]$, platelet distribution width [15], and platelet-to-leukocyte ratio [16] have recently been reported as independent risk factors for CVD. Only a few studies have investigated lymphocyte counts in OSAS patients, with inconsistent results [16, 17]; however, RLC has not been investigated previously in OSAS patients. Therefore, the purpose of this study was to investigate any association between peripheral blood lymphocyte counts and CVD and to assess the predictive value of lymphocyte counts for CVD in patients with OSAS.

\section{Subjects and Methods}

\section{Participants and Clinical Assessment}

The study was conducted at the sleep disorder unit at Farabi Hospital, Faculty of Medicine, Karadeniz Technical University, Trabzon, Turkey, a primary referral center for patients with suspected OSAS. The Institutional Ethics Committee approved the study, and written informed consent was obtained from the participants. One hundred and forty-one subjects aged over 18 years and diagnosed with OSAS using full-night diagnostic polysomnography (PSG) were included in the study. The exclusion criteria were central sleep apnea, Cheyne-Stokes respiration or upper airway resistant syndrome at PSG ( $>20 \%$ events), malignancy, known hematological disease (such as leukemia or myelodysplastic syndrome), liver or kidney disease, chronic alcoholism, or a history of recent blood transfusion (within the previous 2 weeks). Demographic characteristics including age, sex, weight, height, current cigarette smoking status, sleep and medical history, including preexisting diseases diagnosed by a specialist physician, and the use of medication were elicited using our clinic's routine and standardized questionnaire. CVD was defined as heart failure, coronary artery disease, or arrhythmia. All patients were evaluated by a cardiologist (Kayıhan Karaman).

BMI $>30$ was defined as obese. Patients were examined using 2-dimensional pulsed-wave Doppler echocardiography on Vivid 7 (GE Vingmed Ultrasound, Horten, Norway) with a $2.5-\mathrm{MHz}$ transducer for pulmonary artery pressure. Peak systolic pulmonary artery pressure was calculated using the maximal velocity of tricuspid regurgitation on echocardiography.

\section{Polysomnography}

All patients underwent overnight PSG in the sleep unit. All variables, including electroencephalography, bilateral electro-oculography, submental electromyography, thoracic and abdominal movements, saturation of oxyhemoglobin, air flow, electrocardiography, snoring, and video monitoring, were recorded on a commercially available computer system (ALICE Sleepware, Respironics Inc.). Thoracic and abdominal movements were measured with uncalibrated inductive plethysmography, saturation of oxyhemoglobin via finger oximeter, air flow through the nose and mouth via thermistors, and video monitoring via an infrared video camera. The entire recording procedure was performed and observed by experienced sleep technicians. The PSG records were scored in 30-second periods of sleep, breathing, and oxygenation. Hypopnea was defined as a reduction in airflow of $\geq 30 \%$ (recorded by 1 of 3 respiratory signals: a nasal pressure transducer, an oronasal thermal flow sensor, or respiratory inductance plethysmography) lasting $\geq 10 \mathrm{~s}$ with an associated decrease of $\geq 3 \%$ in oxygen saturation or arousal. Apnea was defined as complete cessation ( $\geq 90 \%$ of pre-event baseline) of airflow recorded with an oronasal thermal sensor lasting $\geq 10 \mathrm{~s}$ [18].

Mean numbers of apneas and hypopneas per hour of sleep were calculated as the apnea-hypopnea index (AHI). The diagnosis of sleep-related breathing disorders was based on the International Classification of Sleep Disorders - Second Edition criteria. Patients with AHI $\geq 5$ events per hour and daytime symptoms, such as sleepiness, fatigue, or poor concentration attributable to disrupted sleep were diagnosed as having OSAS [19]. Patients were classified on the basis of the AHI. Subjects with AHI $\geq 5$ events per hour were assigned to the mild OSAS group $(n=26)$, those with $15-30$ events per hour were assigned to the moderate OSAS group $(n=32)$, and those with $>30$ events per hour were assigned to the severe OSAS group $(n=83)$. Twenty-six of the 141 patients enrolled in the study constituted the mild OSAS group, 32 the moderate OSAS group, and the remaining 83 the severe OSAS group. Excessive daytime sleepiness was assessed according to the Epworth Sleepiness Scale (ESS), and values over 10 were regarded as pathological [20].

\section{Laboratory Analysis}

Blood samples were obtained at the first visit following diagnostic PSG and patient enrollment, usually between 8:00 and 10:00 a.m. CBC parameters were determined using a Beckman Coulter LH-750 Hematology Analyzer (Beckman Coulter Inc., Fullerton, Calif., USA). The normal reference value for RLC in our hospital laboratory is between 20.5 and $45.5 \%$.

\section{Statistical Analysis}

The Kolmogorov-Smirnov test was used to determine the normal distribution of continuous variables. Data characterized by a normal distribution are expressed as the mean value and standard deviation. Parameters without such distribution were expressed as medians with ranges. Student's $t$ and Mann-Whitney tests were used to compare the two groups based on the presence or absence of CVD. One-way analysis of variance (ANOVA) was used for parametric variables and Kruskal-Wallis analysis was used for nonparametric variables in order to compare the mild, moderate, and severe OSAS groups. Discrete variables were compared using Fisher's exact test ( $\chi^{2}$ test).

In order to estimate all the potential risk factors for CVD in OSAS patients, multivariate regression analysis was used as a step- 
Table 1. Demographic and clinical characteristics of the study groups

\begin{tabular}{|c|c|c|c|c|}
\hline & $\begin{array}{l}\text { Mild OSAS } \\
(n=26)\end{array}$ & $\begin{array}{l}\text { Moderate OSAS } \\
(\mathrm{n}=32)\end{array}$ & $\begin{array}{l}\text { Severe OSAS } \\
(\mathrm{n}=83)\end{array}$ & $\mathrm{p}$ for trend \\
\hline Age, years & $50.30 \pm 1.49$ & $53.1 \pm 1.12$ & $52.2 \pm 8.53$ & 0.688 \\
\hline Gender (women/men) & $10 / 16$ & $12 / 20$ & $24 / 59$ & 0.602 \\
\hline BMI & $33.99 \pm 9.55$ & $30.98 \pm 6.95$ & $34.14 \pm 7.12$ & 0.202 \\
\hline Smoking & $9(34.6)$ & $7(21.9)$ & $25(30.1)$ & 0.285 \\
\hline \multicolumn{5}{|l|}{ PSG study results } \\
\hline Desaturation index & $9.9 \pm 9.15$ & $12.97 \pm 13.50$ & $50.65 \pm 27.36$ & $<0.001^{*}$ \\
\hline AHI, events/h & $10.39 \pm 3.12$ & $17.56 \pm 7.67$ & $41.48 \pm 20.28$ & $<0.001^{*}$ \\
\hline Minimum oxygen saturation & $86(83-92)$ & $87(83-91)$ & $70(49-83)$ & $<0.001^{*}$ \\
\hline Mean oxygen saturation & $93(91-94)$ & $94(91-94)$ & $87(80-92)$ & $<0.001^{*}$ \\
\hline ESS & $7.4 \pm 4.84$ & $7.56 \pm 4.85$ & $8.54 \pm 5.29$ & 0.596 \\
\hline \multicolumn{5}{|l|}{ Comorbidity } \\
\hline Hypertension & $14(56)$ & $27(71)$ & $47(63)$ & 0.504 \\
\hline Diabetes mellitus & $1(4)$ & $2(7)$ & $10(13)$ & 0.305 \\
\hline CVD & $6(24)$ & $4(13)$ & $13(17)$ & 0.553 \\
\hline \multicolumn{5}{|l|}{ Laboratory variables } \\
\hline Platelet/lymphocyte ratio & $108 \pm 34$ & $120 \pm 35$ & $118 \pm 38$ & 0.414 \\
\hline $\mathrm{MPV}, \mathrm{fl}$ & $8.3 \pm 1.1$ & $8.2 \pm 0.8$ & $8.3 \pm 1.0$ & 0.979 \\
\hline Platelet, $10^{3} / \mu \mathrm{l}$ & $249 \pm 61$ & $259 \pm 52$ & $254 \pm 64$ & 0.598 \\
\hline Leukocyte, $10^{3} / \mu \mathrm{l}$ & $7.7 \pm 1.4$ & $6.7 \pm 1.2$ & $7.6 \pm 1.5$ & 0.132 \\
\hline Lymphocyte, $10^{3} / \mu \mathrm{l}$ & $2.5 \pm 0.6$ & $2.4 \pm 0.5$ & $2.4 \pm 0.7$ & 0.236 \\
\hline Neutrophil, $10^{3} / \mu \mathrm{l}$ & $33.1 \pm 7.2$ & $33.8 \pm 6.7$ & $32.0 \pm 8.1$ & 0.506 \\
\hline $\mathrm{RDW}, \%$ & $13.6 \pm 1.1$ & $13.8 \pm 2.0$ & $14.2 \pm 1.8$ & 0.311 \\
\hline Hemoglobin, g/dl & $14.25 \pm 1.73$ & $13.86 \pm 1.49$ & $14.65 \pm 1.76$ & 0.076 \\
\hline
\end{tabular}

Values represent means $\pm \mathrm{SD}, \mathrm{n}(\%)$, or median (range). ${ }^{*}$ Represents significance.

wise descending method from prognostic factors with a $\mathrm{p}$ value significance of $<0.1$ at univariate analysis. The final regression model included age, RLC, and RDW; $\mathrm{p}<0.05$ was considered statistically significant. Data were analyzed using SPSS statistical software (version 13.01, serial No. 9069728; SPSS Inc., Chicago, Ill., USA).

\section{Results}

One hundred forty-one patients, 95 male and $46 \mathrm{fe}-$ male, with a mean age $51 \pm 8.7$ years were included in the study. Patients' demographic and clinical characteristics and CBC parameters are summarized in table 1 . The desaturation index (number of desaturation episodes per hour) was significantly different between the groups ( $\mathrm{p}<$ 0.001 ). The median AHI was 37 events per hour (range: 19-63). There were no significant differences in terms of the presence of CVD $(\mathrm{p}=0.553)$, hypertension $(\mathrm{p}=$ $0.305)$, or diabetes mellitus $(\mathrm{p}=0.504)$ between the mild, moderate, and severe OSAS groups. There was also no significant difference between the groups in terms of CBC parameters, including platelet $(\mathrm{p}=0.598)$, neutrophil $(\mathrm{p}=0.506)$, leukocyte $(\mathrm{p}=0.132)$, and lymphocyte counts $(\mathrm{p}=0.236) ; \operatorname{RDW}(\mathrm{p}=0.311)$; MPV ( $\mathrm{p}=0.979)$, and platelet-to-lymphocyte ratio $(\mathrm{p}=0.414)$. However, a significant difference was observed in terms of all CBC parameters between patients with an AHI higher or lower than the median value.

Of the 141 patients enrolled, 23 (16.3\%) had CVD. Echocardiographic evaluation was performed on 43 OSAS patients, 11 (25.6\%) of whom had elevated pulmonary artery pressure, and $2(0.5 \%)$ had an ejection fraction $<50$.

The comparison of OSAS patients with or without CVD revealed that patients without CVD were younger than the patients with CVD (mean age: $45 \pm 11.7$ and 53 \pm 11.1 years, respectively; $\mathrm{p}<0.001)$. There were no significant differences in terms of gender, smoking status, or BMI between the groups $(\mathrm{p}=0.414, \mathrm{p}=0.395$, and $\mathrm{p}=$ 0.174 , respectively). Absolute and relative lymphocyte counts were significantly lower in patients with CVD 
Table 2. Comparison of hematological parameters between OSAS patients with and without CVD

\begin{tabular}{lccc}
\hline & $\begin{array}{c}\text { OSAS patients } \\
\text { without CVD }(\mathrm{n}=118)\end{array}$ & $\begin{array}{c}\text { OSAS patients } \\
\text { with CVD }(\mathrm{n}=23)\end{array}$ & p value \\
\hline Hemoglobin, g/dl & $14.5 \pm 1.62$ & $14.0 \pm 2.61$ & 0.263 \\
Platelet, $10^{3} / \mu \mathrm{l}$ & $264.7 \pm 59.3$ & $247.4 \pm 67.4$ & 0.218 \\
MPV, fl & $8.2 \pm 0.987$ & $8.4 \pm 0.99$ & 0.785 \\
Platelet/lymphocyte ratio & $114 \pm 37.6$ & $125.4 \pm 33.8$ & 0.432 \\
RDW, \% & $13.4(13.1-4.6)$ & $14.2(13.4-17.5)$ & $0.005^{*}$ \\
Leukocyte, $10^{3} / \mu \mathrm{l}$ & $7.3 \pm 1.3$ & $7.4 \pm 1.8$ & 0.611 \\
Neutrophil, $10^{3} / \mu \mathrm{l}$ & $4.6 \pm 1.5$ & $4.3 \pm 1.6$ & 0.303 \\
Neutrophil, $\%$ & $58.5 \pm 9.2$ & $56.6 \pm 8.2$ & 0.230 \\
Lymphocyte, $10^{3} / \mu \mathrm{l}$ & $2.5 \pm 0.6$ & $2.0 \pm 0.6$ & $0.004^{*}$ \\
Lymphocyte, $\%$ & $33.9 \pm 7.5$ & $28.3 \pm 6.7$ & $0.001^{*}$ \\
\hline
\end{tabular}

Values represent means \pm SD or median (range). ${ }^{*}$ Indicates significance.

Table 3. Independent predictors of CVD determined by multivariate linear regression analysis in patients with OSAS (multivariate analysis)

\begin{tabular}{llrl}
\hline & Odds ratio & $95 \%$ CI & p value \\
\hline Age & 1.1 & $1.0-1.2$ & 0.002 \\
RDW & 1.5 & $1.1-2.0$ & 0.012 \\
RLC & 0.9 & $0.85-1.0$ & 0.042 \\
\hline
\end{tabular}

than in patients without CVD (mean absolute lymphocyte counts: $2.0 \times 10^{3}$ vs. $2.5 \times 10^{3} \mu \mathrm{l}, \mathrm{p}=0.004$; mean RLC: 28.3 vs. $33.9 \%, p=0.001$, respectively) (table 2 ). RDW was also significantly higher in patients with CVD (13.4) than the mean value for patients without CVD (14.2) ( $\mathrm{p}=$ $0.005)$. There were no significant differences in terms of the other parameters (table 2).

The results of multiple regression analysis showed that age (odds ratio $=1.1,95 \% \mathrm{CI}: 1.0-1.2, \mathrm{p}=0.002$ ), RLC (odds ratio $=0.9,95 \%$ CI: 0.85-1.0, $\mathrm{p}=0.042$ ), and RDW (odds ratio $=1.5,95 \%$ CI: $1.1-2.0, \mathrm{p}=0.012$ ) were independently associated with the presence of CVD in patients with OSAS (table 3).

\section{Discussion}

In this study, absolute and relative lymphocyte counts were similar in patients with mild, moderate, or severe OSAS and were not related to the severity of OSAS. The RDW was significantly higher in OSAS patients with CVD than without and independently associated with the presence of CVD. Additionally, RLC was also independently associated with the presence of CVD in patients with OSAS. A significant association was found between RLC and the presence of CVD in OSAS patients, but not absolute lymphocyte count. This could be due to a shift in leukocyte differentials resulting in the decrease of the lymphocyte level without any change in total leukocyte count.

Our findings indicated that low RLC is significant only in the presence of CVD in patients with OSAS; however, there was no association between OSAS severity and lymphocyte count, similar to the findings of Freire et al. [17] but not that of Koseoglu et al. [16]. One probable explanation could be that in these studies; analysis was performed without considering the presence of RLC or CVD, unlike our study, in which the presence of RLC and CVD contributed to the significance of the lymphocyte count. Freire et al. [17] investigated the relationship between white blood cells and lymphocytes, neutrophil and total leukocyte counts, and AHI, but identified no differences. However, they did not analyze RLC and did not consider comorbidities in patients with OSAS, including CVD. Koseoglu et al. [16] observed a significantly lower lymphocyte count in patients with OSAS compared to the controls, and reported that the decrease was most prominent in the severe OSAS group. However, they also reported significant differences between the study groups in terms of comorbidities, including CVD, which may account for the significant low lymphocyte count. Additionally, they did not analyze the significance of the lymphocyte count in OSAS patients with or without CVD and did not investigate the significance of RLC. 
In our study, there was no association between MPV, severity OSAS, and the presence of CVD. Other studies $[12,13]$, however, have reported a correlation between the severity of OSAS and MPV, although Sökücü et al. [21] did not report such an association in nondiabetic, nonischemic OSAS patients. Considering this discrepancy in the results, in contrast to our study Varol et al. [12] did not consider comorbidities including CVD that are frequently reported in cases of severe OSAS. Kanbay et al. [13] reported a significantly higher CVD rate in the severe OSAS group, which may have affected their findings of high serum MPV values in patients with severe and moderate OSAS, but not in the mild OSAS group.

Probable mechanisms have been suggested for patients with OSAS including oxidative stress inducing systemic cortisol production and hypothalamic-pituitaryadrenal axis dysfunctions that could interact with lymphocyte count in patients with OSAS [22-24]. Equally important, the investigation of lymphocyte subtypes could also be essential for identifying the mechanism involved. Several studies [25] investigating lymphocyte subpopulations in patients with OSAS have indicated the involvement of lymphocytes in the pathogenic mechanism of OSAS. Total lymphocyte and CD4+ lymphocyte counts were significantly decreased in patients with OSAS using CPAP therapy $>4 \mathrm{~h}$ per night, and were still evident after 6 months of adherent CPAP use [25]. Ye et al. [26] recently reported that the balance between newly discovered subsets of $\mathrm{T}$ helper cells, Th17 and $\mathrm{T}$ regulatory (Treg), is impaired in patients with OSAS by demonstrating an increase in peripheral Th17 and a decrease in Treg levels. Th17 and Treg cells have opposite effects on autoimmunity and inflammation. Although these studies in- dicate the involvement of lymphocytes in the pathophysiological mechanism associated with OSAS, the results are still preliminary and require confirmation.

The limitations to this study were its cross-sectional design which made it difficult to draw inferences regarding the causal relationships between RLC and OSAS, nonevaluation of subtype of peripheral lymphocytes, failure to do no follow-up (hence the prognostic outcomes of low RLC on CVD in patients with OSAS were not available), and failure to investigate the effect of continuous positive nasal airway pressure treatment on RLC.

\section{Conclusion}

In this study, lymphocyte counts were associated with CVD but not with the severity of OSAS. RLC, a widely available diagnostic tool with no additional costs, could be used as a biomarker for evaluating CVD in patients with OSAS.

\section{Acknowledgments}

PSG recordings were performed by Jale Ayyıldı, Ayse Duman, Hüseyin Adanur, Zeki Ertem, Pelin Alev, Umit Dincel, and Yildiray Yigici. The authors thank all of the PSG technicians. The authors also thank Dr. Kayıhan Karaman who performed the cardiological evaluation.

\section{Disclosure Statement}

The authors report no conflicts of interest.

\section{References}

-1 Fava C, Montagnana M, Favaloro EJ, et al: Obstructive sleep apnea syndrome and cardiovascular diseases. Semin Thromb Hemost 2011;37:280-297.

-2 Ryan S, Taylor CT, McNicholas WT: Systemic inflammation: a key factor in the pathogenesis of cardiovascular complications in obstructive sleep apnoea syndrome? Thorax 2009;64:631-636.

$\checkmark 3$ Buttarello M, Plebani M: Automated blood cell counts: state of the art. Am J Clin Pathol 2008;130:104-116.

4 Hong LF, Li XL, Luo SH, et al: Relation of leukocytes and its subsets counts with the severity of stable coronary artery disease in patients with diabetic mellitus. PLoS One 2014;9: e90663.
5 Icli A, Tayyar S, Varol E, et al: Mean platelet volume is increased in infective endocarditis and decreases after treatment. Med Princ Pract 2013;22:270-273.

6 Sahin O, Akpek M, Sarli B, et al: Association of red blood cell distribution width levels with severity of coronary artery disease in patients with non-ST elevation myocardial infarction. Med Princ Pract 2015;24:178-183.

7 Balta S, Ozturk C: The platelet-lymphocyte ratio: a simple, inexpensive and rapid prognostic marker for cardiovascular events. Platelets 2014;30:1-2.

$>8$ Nacar AB, Erayman A, Kurt M, et al: The relationship between coronary collateral circulation and neutrophil/lymphocyte ratio in patients with coronary chronic total occlusion. Med Princ Pract 2015;24:65-69.
-9 Vaduganathan M, Ambrosy AP, Greene SJ, et al: Predictive value of low relative lymphocyte count in patients hospitalized for heart failure with reduced ejection fraction: insights from the EVEREST trial. Circ Heart Fail 2012;5: $750-758$.

10 Boag SE, Das R, Shmeleva EV, et al: T lymphocytes and fractalkine contribute to myocardial ischemia/reperfusion injury in patients. J Clin Invest 2015;125:3063-3076.

11 Milo-Cotter O, Felker GM, Uriel N, et al: Patterns of leukocyte counts on admissions for acute heart failure - presentation and outcome - results from a community based registry. Int J Cardiol 2011;148:17-22. 
12 Varol E, Ozturk O, Gonca T, et al: Mean platelet volume is increased in patients with severe obstructive sleep apnea. Scand J Clin Lab Invest 2010;70:497-502.

13 Kanbay A, Tutar N, Kaya E, et al: Mean platelet volume in patients with obstructive sleep apnea syndrome and its relationship with cardiovascular diseases. Blood Coagul Fibrinolysis 2013;24:532-536.

14 Ozsu S, Abul Y, Gulsoy A, et al: Red cell distribution width in patients with obstructive sleep apnea syndrome. Lung 2012;190:319326.

15 Kurt OK, Yildiz N: The importance of laboratory parameters in patients with obstructive sleep apnea syndrome. Blood Coagul Fibrinolysis 2013;24:371-374.

16 Koseoglu HI, Altunkas F, Kanbay A, et al: Platelet-lymphocyte ratio is an independent predictor for cardiovascular disease in obstructive sleep apnea syndrome. J Thromb Thrombolysis 2015;39:179-185.

17 Freire AX, Kadaria D, Avecillas JF, et al: Obstructive sleep apnea and immunity: rela- tionship of lymphocyte count and apnea hypopnea index. South Med J 2010;103:771774.

18 Berry RB, Budhiraja R, Gottlieb DJ, et al; American Academy of Sleep Medicine: Rules for scoring respiratory events in sleep: update of the 2007 AASM Manual for the Scoring of Sleep and Associated Events. Deliberations of the Sleep Apnea Definitions Task Force of the American Academy of Sleep Medicine. J Clin Sleep Med 2012;8:597-619.

19 American Academy of Sleep Medicine: International Classification of Sleep Disorders: Diagnostic and Coding Manual, ed 2. Westchester, AASM, 2005, pp 51-55.

20 Johns MW: A new method for measuring daytime sleepiness: the Epworth Sleepiness Scale. Sleep 1991;14:540-545.

21 Sökücü SN, Ozdemir C, Dalar L, et al: Is mean platelet volume really a severity marker for obstructive sleep apnea syndrome without comorbidities? Pulm Med 2014:754839.

22 Vgontzas AN, Pejovic S, Zoumakis E, et al: Hypothalamic-pituitary-adrenal axis activity in obese men with and without sleep apnea: effects of continuous positive airway pressure therapy. J Clin Endocrinol Metab 2007;92: 4199-4207.

23 Dadoun F, Darmon P, Achard V, et al: Effect of sleep apnea syndrome on the circadian profile of cortisol in obese men. Am J Physiol Endocrinol Metab 2007;293:466-474.

24 Carneiro G, Togeiro SM, Hayashi LF, et al: Effect of continuous positive airway pressure therapy on hypothalamic-pituitary-adrenal axis function and 24-h blood pressure profile in obese men with obstructive sleep apnea syndrome. Am J Physiol Endocrinol Metab 2008;295:380-384.

25 Steiropoulos P, Kotsianidis I, Nena E, et al: Long-term effect of continuous positive airway pressure therapy on inflammation markers of patients with obstructive sleep apnea syndrome. Sleep 2009;32:537-543.

26 Ye J, Liu H, Zhang G, et al: The Treg/Th17 imbalance in patients with obstructive sleep apnoea syndrome. Mediators Inflamm 2012: 815308 . 